hep-ph/9707276

CERN-TH/97-153

DFTT $41 / 97$

Edinburgh $97 / 8$

UB-ECM-PF 97/11

\title{
WHAT CAN WE LEARN FROM POLARIZED STRUCTURE FUNCTION DATA?
}

\author{
Richard D. Ball, ${ }^{\star}$ Giovanni Ridolfi,* \\ Guido Altarelli ${ }^{\dagger}$ and Stefano Forte ${ }^{\ddagger}$ \\ ${ }^{\star}$ Department of Physics and Astronomy, 由 \\ University of Edinburgh, Scotland \\ *INFN, Sezione di Genova, \\ Via Dodecaneso 33, I-16146 Genova, Italy \\ †Theoretical Physics Division, CERN, \\ CH-1211 Geneva 23, Switzerland \\ † Terza Università di Roma, Rome, Italy \\ ${ }^{\ddagger}$ INFN, Sezione di Torino, \\ Via P. Giuria 1, I-10125 Torino, Italy \\ ${ }^{\ddagger}$ Departament ECM, Universitat de Barcelona, \\ Diagonal 647, E-08028 Barcelona, Spain
}

\begin{abstract}
We summarise the perturbative QCD analysis of the structure function data for $g_{1}$ from longitudinally polarized deep inelastic scattering from proton, deuteron and neutron targets, with particular emphasis on testing sum rules, determining helicity fractions, and extracting the strong coupling from both scaling violations and the Bjorken sum rule.
\end{abstract}

Talk at Les Rencontres de Physique de la Valle d'Aoste, La Thuile, March 1997, and at DIS9\%, Chicago, April 1997

to be published in the proceedings

June 1997

\footnotetext{
${ }^{1}$ Royal Society University Research Fellow

${ }^{2}$ IBERDROLA visiting professor
} 
The data on the nucleon structure function $g_{1}$, determined from measurements of the polarization asymmetry in longitudinally polarized deep inelastic scattering, is now sufficient to justify a sophisticated NLO analysis using the full machinery of perturbative QCD [1]. It is possible to test sum rules, extract polarized parton distributions and determine parton helicity fractions, and even, as we shall see, make a reasonably accurate determination of $\alpha_{s}$. Important issues in these analyses are the dependence on the functional forms used to parameterize the initial parton distributions, and, when estimating first moments, the implications for the extrapolation to small $x$. Here we will discuss the results obtained in a recent analysis [2].

In perturbative QCD structure functions are decomposed into convolutions of perturbatively calculable coefficient functions and intrinsically nonperturbative parton distribution, which then vary with $Q^{2}$ according to perturbative evolution equations [3]. For $g_{1}$ the appropriate partons are [4, 5] the nonsinglet and singlet polarized sea quark distributions, $\Delta q^{N S}\left(x, Q^{2}\right)$ and $\Delta q^{S}\left(x, Q^{2}\right)$ (where $\Delta q \equiv q^{\uparrow}-q^{\downarrow}+\bar{q}^{\uparrow}-\bar{q}^{\downarrow}$ ), and the polarized gluon distribution $\Delta g\left(x, Q^{2}\right) \equiv g^{\uparrow}-$ $g^{\downarrow}$. Under evolution $\Delta q^{S}$ and $\Delta g$ mix, while $\Delta q^{N S}$ evolves independently. Valence distributions (of the form $q^{\uparrow}-q^{\downarrow}-\left(\bar{q}^{\uparrow}-\bar{q}^{\downarrow}\right)$ ) also evolve independently, but cannot be measured in inclusive scattering of virtual photons, and are thus not accessible from data on $g_{1}$ alone (unless of course extra assumptions are made about the relative size of valence and sea, as in ref.[6, 7]). The coefficient functions $C_{q}^{N S}, C_{q}^{S}$ and $C_{g}$ and splitting functions $P_{q q}^{N S}, P_{q q}^{S}, P_{q g}, P_{g q}$ and $P_{g g}$ are now all available at NLO [8].

First moments of polarized parton distribution functions are of special interest, since they have a direct interpretation as the helicity fractions of the various constituents. First moments of nonsinglet distributions are chosen to be independent of $Q^{2}$ (PCAC), and may then be related to nonsinglet axial charges $\left(g_{A} \equiv a_{3}\right.$ and $a_{8}$ for the $S U(2)$ triplet and $S U(3)$ octet) measured in weak baryon decays. The first moment of the singlet distribution $\Delta \Sigma \equiv \int_{0}^{1} d x \Delta q^{S}$ may also be chosen to be independent of $Q^{2}$ : this is a direct consequence of the Adler-Bardeen theorem. The singlet axial charge is then

$$
a_{0}\left(Q^{2}\right)=\Delta \Sigma-n_{f} \frac{\alpha_{s}\left(Q^{2}\right)}{2 \pi} \Delta g\left(Q^{2}\right)
$$

It evolves multiplicatively, beginning at two loops, because of the axial anomaly: this implies that $\Delta g\left(Q^{2}\right)$ grows as $\log Q^{2}$, and that $a_{0}(\infty)$ and $\Delta \Sigma$ will in general be very different [9]. Changes in factorization scheme away from these 'AB schemes' 50 result in only small changes in $\Delta g$ (of $\left.O\left(\alpha_{s}\right)\right)$, but large changes in $\Delta \Sigma$ (which however is then no longer scale independent).

Sum rules express the first moments of nonsinglet and singlet combinations of structure functions $g_{1}$ in terms of nonsinglet and singlet axial charges:

$$
\int_{0}^{1} d x g_{1}^{N}\left(x, Q^{2}\right)=\frac{1}{18}\left[\left(1-c^{N S}\right)\left(3 \epsilon a_{3}+a_{8}\right)+\left(1-c^{S}\right) 4 a_{0}(\infty)\right],
$$

where $\epsilon=+1,0,-1$ for $\mathrm{N}=\mathrm{p}, \mathrm{d}, \mathrm{n}$ respectively. The isospin triplet (Bjorken) sum rule, for example, relates the first moment of $g_{1}^{p}-g_{1}^{n}$ to $g_{A}$. The perturbative corrections $c^{N S}$ and $c^{S}$, which begin at NLO (i.e. at $O\left(\alpha_{s}\right)$ ), are now both known at NNNLO (i.e. to $O\left(\alpha_{s}^{3}\right)$ ) [10].

In order to compute first moments of parton distributions and structure functions it is necessary to extrapolate the data in the measured region down to $x=0$. The behaviour of $F_{2}^{p}$ at small $x$ is now well understood due to data from HERA [11]: while at low $Q^{2} x q^{S}\left(x, Q^{2}\right)$ is fairly flat, in accordance with Regge theory since the pomeron intercept is close to unity, as $Q^{2}$ 
increases it rises more and more steeply due to a rise in the gluon distribution $x g\left(x, Q^{2}\right)$ driven by gluon bremsstrahlung [12]. By contrast Regge theory predicts that $x q^{N S}\left(x, Q^{2}\right)$ decreases rapidly at small $x$ (so $q^{N S}\left(x, Q^{2}\right) \ll q^{S}\left(x, Q^{2}\right)$ ), though with a rise in $q^{N S}\left(x, Q^{2}\right)$ which is then stable under perturbative evolution. Now according to Regge theory $\left|\Delta q^{N S}\left(x, Q^{2}\right)\right|$ and $\left|\Delta q^{S}\left(x, Q^{2}\right)\right|$ should be flat (or falling) at small $x$ and $Q^{2}$, and perturbative evolution then leads to an instability in both distributions 13 . However $\Delta q^{S}$ and $\Delta g$ mix nontrivially, and the eigenvectors of their evolution are such that they eventually have opposite sign. As a consequence although $\Delta q^{N S}\left(x, Q^{2}\right)$ and $\Delta g\left(x, Q^{2}\right)$ both rise at small $x$ and increasing $Q^{2}$, $\Delta q^{S}\left(x, Q^{2}\right)$ falls steeply and $g_{1}^{N}\left(x, Q^{2}\right)$ is eventually driven negative [4]. Estimates of small $x$ contributions using Regge extrapolation alone will then be unreliable, underestimating their size and sometimes even giving them the wrong sign.

Here our general philosophy will be to estimate small $x$ contributions by performing fits to the data which are consistent with Regge behaviour at small $x$ and low $Q^{2}$, but have the effects of perturbative evolution superimposed at higher $Q^{2}$. Our general procedure is the standard one: we take some initial parameterization of $\Delta q^{N S}\left(x, Q_{0}^{2}\right), \Delta q^{S}\left(x, Q_{0}^{2}\right)$ and $\Delta g\left(x, Q_{0}^{2}\right)$, evolve these three distributions to the $Q^{2}$ of the data [14, 15, 16, 17] using NLO perturbative evolution (in the AB scheme), compute $g_{1}\left(x, Q^{2}\right)$ using the NLO coefficient functions, and then adjust the parameters in order to optimize the fit. The quality of such a fit may be judged from the figure. In principle it should be possible to fit both triplet and octet nonsinglet distributions (the charm and bottom distributions are generated radiatively from threshold), but in practice this is not possible with present data since the triplet dominates $\left(a_{8} \ll 3 a_{3}\right)$. We thus assume that both distributions have the same shape, and find that the results of the fits are very insensitive to the value of $a_{8}$.

In such fits it is important to assess the dependence of the results on the form of the initial parameterization, otherwise errors may be underestimated. This is particularly true here, where we want to compute first moments, since the shape of the small $x$ tails may depend very much on the parameterization adopted. To this end we tried four different parameterizations:

A: the standard form with powers of $x$ and $1-x$, and $Q_{0}^{2}=1 \mathrm{GeV}^{2}$;

B: powers of $\ln 1 / x$ and $Q_{0}^{2}=1 \mathrm{GeV}^{2}$;

C: as A, but with $Q_{0}^{2}=0.3 \mathrm{GeV}^{2}$ and falling inputs;

D: as C, but with valencelike Regge behaviour fixed by hand.

All four parameterizations give fits to the data of comparable quality. Furthermore they all lead to structure functions which eventually become negative at small $x$ (see figure), as expected. However the size of the small $x$ contribution varies quite widely between the four distributions, and we take this spread as an estimate of the error on the small $x$ extrapolation. It follows that our first moments for $g_{1}^{p}, g_{1}^{d}$ and $g_{1}^{n}$ will all be smaller, but with a larger error, than those obtained using Regge extrapolations (see table 1). By contrast our first moment of $g_{1}^{p}-g_{1}^{n}$ is larger, since the nonsinglet distribution rises at small $x$.

Fixing $\alpha_{s}\left(m_{Z}\right)=0.118 \pm 0.005[18]$, and, very conservatively, $a_{8}=0.6 \pm 0.2$, we may use the fits to determine $g_{A}, \Delta \Sigma, \Delta g$ and $a_{0}$. The results are shown in table 2 . The theoretical error includes the uncertainty in the small $x$ extrapolation (underestimated in ref.[0]), and the effect of higher order corrections estimated by variation of the renormalization and factorization scales (not included in ref.[7]). Although the shapes of the distributions are not fixed very precisely by the data, their first moments are surprisingly well determined. Fitting $g_{A}$ is a test of the 
Bjorken sum rule: the value obtained from $\beta$-decay is 1.26 , so we verify the Bjorken sum rule at the $8 \%$ level. Similarly fitting $\Delta \Sigma$ may be regarded as a test of the constituent quark inspired ansatz $\Delta \Sigma \approx a_{8}$ : again this is confirmed to within one standard deviation (though it must be remembered that such a test is only meaningful in $\mathrm{AB}$ schemes; in other schemes $\Delta \Sigma$ is scale dependent and may take any value one chooses). As was predicted in ref. [9], a large positive value of $\Delta g\left(1 \mathrm{GeV}^{2}\right)$ (two standard deviations from zero) is then responsible for the small value of the singlet axial charge $a_{0}$ (compatible results for $\Delta g$ were also found in [4, 5, 6, 7, 14]). Our extrapolation to small $x$ is particularly important here: Regge extrapolations give a rather larger value for the axial singlet charge, with a smaller (but incorrect) estimate of the error [19, 14]. There is still no totally convincing explanation of why $a_{0}$ should be consistent with zero.

The recently published neutron data now make it possible to include $\alpha_{s}$ in the fit, giving the result

$$
\alpha_{s}\left(m_{Z}\right)=0.120_{-0.005}^{+0.004}(\text { exp. })_{-0.006}^{+0.009}(\text { th. })
$$

This determination is possible because of the scaling violations in the nonsinglet channel, which are not sensitive to uncertainties in the gluon distribution: without the neutron data the error would have been twice as large. It is thus not affected by uncertainties in the small $x$ extrapolation: the dominant error instead comes from higher order corrections, which are particularly large as much of the data is at fairly low $Q^{2}$. It is tempting to attempt to reduce this uncertainty by determining $\alpha_{s}$ from the Bjorken sum rule [20], exploiting the NNNLO calculations of ref. [10]. Unfortunately the relatively large error in the Bjorken sum resulting from the small $x$ extrapolation makes this method impractical at present: we find $\alpha_{s}\left(m_{Z}\right)=$ $0.118_{-0.024}^{+0.010}$.

The test of the Bjorken sum rule, the determination of helicity fractions, and the result for $\alpha_{s}$ would all be improved by data with better statistics at higher $Q^{2}$, by an independent measurement of $\Delta g$ through open charm production [21], or by data at smaller $x$ [22]. A better theoretical understanding of polarized structure functions at small $x$ would also be useful. However since the higher order corrections now involve double logarithms of $x$ [23], rather than the single (and thus factorizable [24]) small $x$ logarithms in the unpolarized case, this may prove to be a considerable challenge. 


\section{References}

[1] G. Ridolfi, in the proceedings of "DIS96", hep-ph/9610214;

S. Forte, in the proceedings of "SPIN96", hep-ph/9610238.

[2] G. Altarelli et al., Nucl. Phys. B496 (1997) 337.

[3] G. Altarelli and G. Parisi, Nucl. Phys. B126 (1977) 298.

[4] R.D. Ball et al., Nucl. Phys. B444 (1995) 287.

[5] R.D. Ball et al., Phys. Lett. B378 (1996) 255;

S. Forte et al., in the proceedings of "DIS96", hep-ph/9608399.

[6] M. Glück et al., Phys. Rev. D53 (1996) 4775;

T. Gehrmann and J. Stirling, Phys. Rev. D53 (1996) 6100.

[7] K. Abe et al., hep-ph/9705344 and P. Zyla, these proceedings.

[8] R. Mertig and W. L. van Neerven, Zeit. Phys. C70 (1996) 637;

W. Vogelsang, Phys. Rev. D54 (1996) 2023.

[9] G. Altarelli and G. G. Ross, Phys. Lett. B212 (1988) 391;

R.D. Carlitz et al., Phys. Lett. B214 (1988) 229.

[10] S. A. Larin et al., Phys. Lett. B259 (1991) 345; hep-ph/9702435.

[11] R.D. Ball and A. DeRoeck, in the proceedings of "DIS96", hep-ph/9609309.

[12] A. De Rújula et al., Phys. Rev. 10 (1974) 1649;

R.D. Ball and S. Forte, Phys. Lett. B335 (1994) 77.

[13] M. A. Ahmed and G. G. Ross, Phys. Lett. B56 (1975) 385.

[14] SMC Collaboration, D. Adams et al., CERN-PPE 97-08, hep-ex/9702005.

[15] E143 Collaboration, K. Abe et al., Phys. Rev. Lett. 74 (1995) 346;

Phys. Lett. B364 (1995) 61.

[16] E142 Collaboration, P.L. Anthony et al., Phys. Rev. D54 (1996) 6620.

[17] E154 Collaboration, K. Abe et al., SLAC-PUB-7459, hep-ex/9705012.

[18] G. Altarelli, in the proceedings of "CRAD96", hep-ph/9611239.

[19] G. Altarelli and G. Ridolfi, Nucl. Phys. B (Proc. Suppl.) 39B (1995) 106.

[20] J. Ellis and M. Karliner, Phys. Lett. B341 (1995) 397.

[21] A. Bravar, these proceedings.

[22] R.D. Ball et al., hep-ph/9609515;

A. DeRoeck, these proceedings.

[23] R. Kirschner and L. Lipatov, Nucl. Phys. B213 (1983) 122;

J. Bartels et al., Zeit. Phys. C70 (1996) 273; C72 (1996) 627.

[24] R.D. Ball and S. Forte, hep-ph/9703417 and in these proceedings. 


\begin{tabular}{|l|c|l|l|}
\hline & $\left\langle Q^{2}\right\rangle$ & Regge extrapolation & pQCD extrapolation \\
\hline SMC $: \mathrm{p}$ & 10 & $0.136 \pm 0.016$ & $0.116 \pm 0.022$ \\
E143 $: \mathrm{p}$ & 3 & $0.127 \pm 0.010$ & $0.107 \pm 0.017$ \\
\hline SMC $: \mathrm{d}$ & 10 & $0.041 \pm 0.007$ & $0.021 \pm 0.016$ \\
E143 $: \mathrm{d}$ & 3 & $0.042 \pm 0.010$ & $0.021 \pm 0.014$ \\
\hline E142 $: \mathrm{n}$ & 2 & $-0.033 \pm 0.011$ & $-0.068 \pm 0.015$ \\
E154 $: \mathrm{n}$ & 5 & $-0.041 \pm 0.007$ & $-0.070 \pm 0.015$ \\
\hline
\end{tabular}

Table 1: Results for complete first moments of $g_{1}^{N}$.

\begin{tabular}{|l|llll|}
\hline & $g_{A}$ & $\Delta \Sigma$ & $\Delta g(1)$ & $a_{0}(\infty)$ \\
\hline Central Value & 1.19 & 0.45 & 1.6 & 0.10 \\
Exp error & 0.05 & 0.04 & 0.4 & 0.05 \\
Th error & 0.07 & 0.08 & 0.8 & ${ }_{-0.10}^{+0.17}$ \\
\hline
\end{tabular}

Table 2: Axial charges and helicity fractions. 

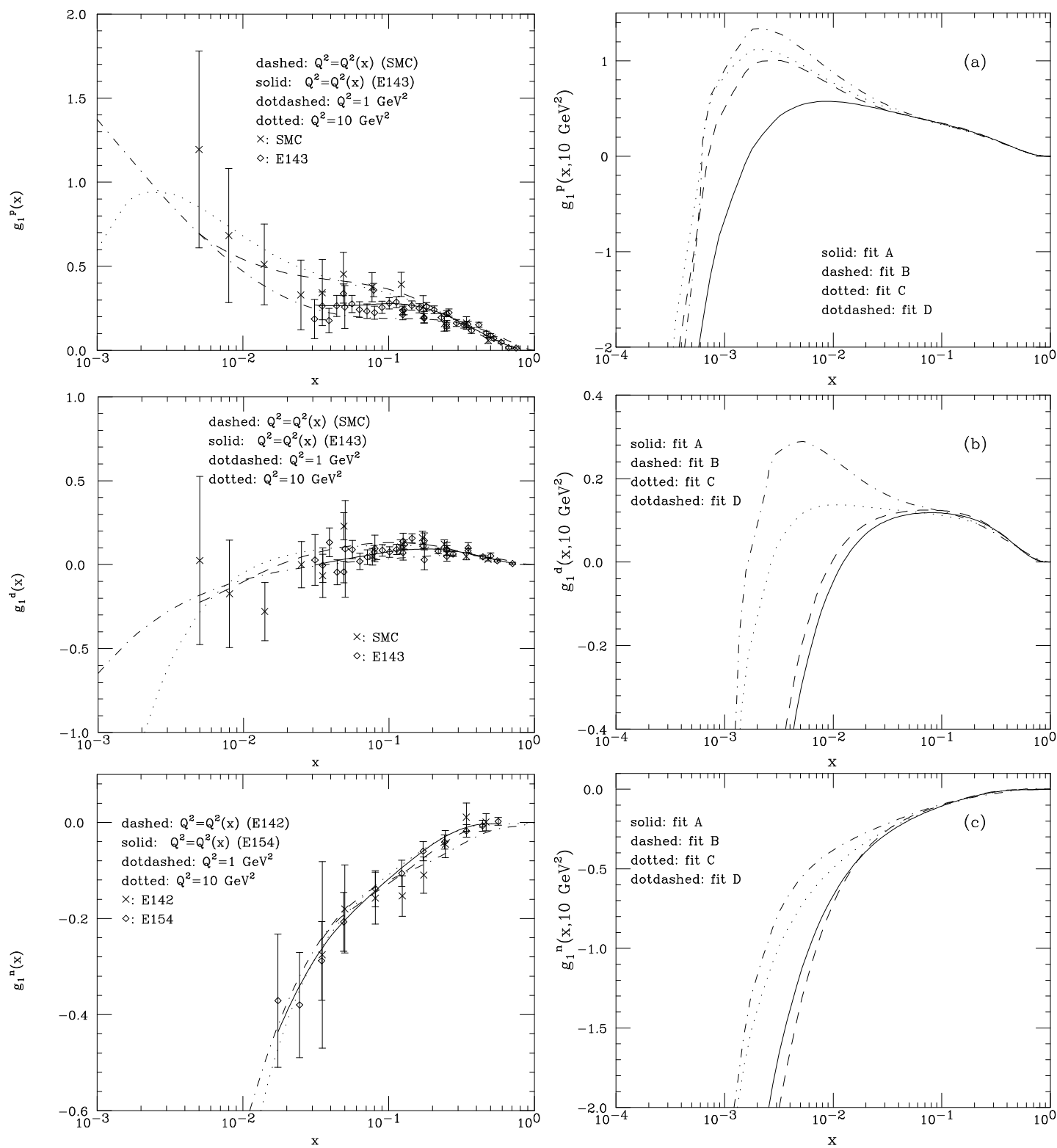

Figure 1: Fits to the data: in the left column we plot $g_{1}^{N}$, and compare fit $B$ to the data, while in the right column we plot $g_{1}^{N}$ at $Q^{2}=10 \mathrm{GeV}^{2}$ for fits $A-D$, showing the differences between the small $x$ tails. Figures a,b and c are for proton, deuteron and neutron respectively. 